\title{
THE PHYSIOLOGICAL DISPOSITION AND METABOLIC FATE OF CORTISONE IN MAN
}

\author{
By RALPH E. PETERSON, CHARLES E. PIERCE, JAMES B. WYNGAARDEN,1 \\ JOSEPH J. BUNIM, AND BERNARD B. BRODIE
}

\author{
(From the National Institute of Arthritis and Metabolic Diseases, and the National Heart \\ Institute, National Institutes of Health, Bethesda, Md.)
}

(Submitted for publication March 15, 1957; accepted May 2, 1957)

In an earlier paper we described studies on the physiological disposition and metabolic fate of hydrocortisone in man (1). This paper describes similar studies on cortisone. Although contributing very little to the body pool of adrenal cortical steroids, cortisone has been used extensively as a therapeutic agent, and it was therefore of interest to compare its metabolism with that of hydrocortisone. Large doses of cortisone and hydrocortisone were administered intravenously and rates of disappearance from the plasma determined in normal subjects and in patients with liver disease, myxedema, thyrotoxicosis, and rheumatoid arthritis. Trace quantities of cortisone-4- $\mathrm{C}^{14}$ and hydrocortisone-4-C ${ }^{14}$ were employed in certain studies. From these studies, information was obtained regarding rate of metabolic transformation, rate of absorption, rates and routes of excretion of cortisone and its metabolites, and, to some extent, the identity and amount of these metabolites. Information was also obtained on the in vivo conversion of cortisone to hydrocortisone.

\section{MATERIALS AND METHODS}

Administration of steroid. The steroids were dissolved in $25 \mathrm{ml}$. ethanol and added to $500 \mathrm{ml}$. of sterile five per cent dextrose in water. This solution was administered intravenously over a period of 10 to 20 minutes. For oral or intramuscular administration the steroids were suspended in saline. Cortisone-4-C $\mathrm{C}^{\mathrm{A}}$ was administered intravenously in trace quantities in a small volume of five per cent ethanol in water over a period of 3 to 5 minutes, or mixed with carrier cortisone and given in $500 \mathrm{ml}$. of three per cent ethanol in five per cent dextrose in sterile distilled water. Following the administration of the steroids, blood samples were drawn in heparinized syringes every 20 or 30 minutes for 2 hours or more. Urine samples were collected and preserved by freezing.

Methods of assay. The extraction procedures for the steroids in plasma and urine were the same as those de-

\footnotetext{
1 Present address : Department of Medicine, Duke University, Durham, North Carolina.
}

scribed previously (1). Cortisone and certain of its metabolites in plasma were determined by a modification (2) of the original phenylhydrazine procedure of Silber and Porter (3). Specificity of this method of assay for plasma hydrocortisone after administration of hydrocortisone was shown to be high, as tested by countercurrent distribution and isotope dilution techniques. Using these same techniques, it was found that the SilberPorter method was much less satisfactory for the specific measurement of cortisone after the administration of cortisone.

Counter-current distribution. Plasma drawn $60 \mathrm{~min}-$ utes after a 20 -minute intravenous infusion of $200 \mathrm{mg}$. of cortisone was extracted with dichloromethane. A trace quantity of cortisone-4-C $\mathrm{C}^{14}$ was added to the dichloromethane extract. The extract was evaporated to dryness, and the apparent cortisone subjected to a 16-transfer counter-current distribution. The solvents used were one volume of water as the upper phase and one volume of a mixture containing four parts carbon tetrachloride and one part dichloromethane as the lower phase. This system gave a partition coefficient of 1.5 for cortisone. After distribution, aliquots of both phases were assayed by the phenylhydrazine reaction, and for carbon ${ }^{14}$. Figure 1 shows the distribution of the phenylhydrazine reacting material in plasma, and the cortisone-4-C $C^{4}$. The data indicate that more than one-half of the dichloromethane-soluble material in plasma reacting with phenylhydrazine was not cortisone. The major metabolites giving the phenylhydrazine reaction are distributed in a manner like hydrocortisone $(K=5.2)$ and tetrahydrocortisone $(\mathrm{K}=4.8)$.

Isotope dilution. The plasma samples obtained following the administration of cortisone were subjected to an isotope dilution assay, and additional aliquots were assayed by the phenylhydrazine procedure. The isotope dilution assay was carried out by first adding a known quantity of cortisone-4-C $C^{16}$ to a known volume of plasma. The plasma was extracted with dichloromethane, and the dichloromethane extract evaporated to dryness in vacuo and subjected to paper chromatography in a modified Bush-type (4) system (cyclohexane 2: benzene $4:$ methanol 4: water 1), along with a cortisone standard. Following development of the chromatogram for 18 to 24 hours, the paper strip was removed and scanned with ultraviolet light. The cortisone reference standard was located and marked (cortisone moves about $20 \mathrm{~cm}$. in 24 hours). The band of paper containing the plasma 


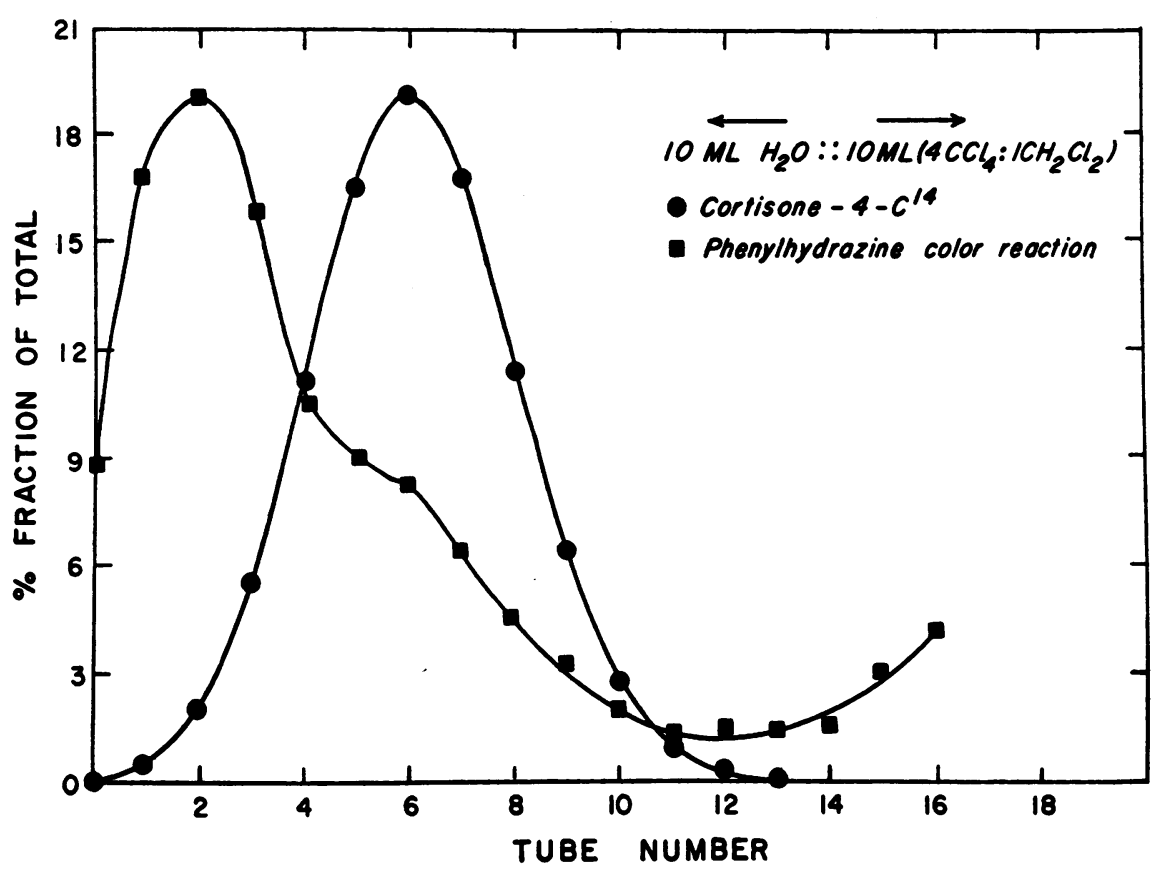

Fig. 1. Counter-Current Distribution of Plasma Steroids Reacting with Phenylhydrazine 60 Minutes After Infusion of 200 mg. Cortisone

cortisone was cut into a very narrow strip, perpendicular to the running direction of the solvent. The cortisone was eluted from this paper strip with $3 \mathrm{ml}$. of 95 per cent cold ethanol and the ethanol evaporated to dryness under a stream of nitrogen.

The residue was dissolved in a small volume of dichloromethane ( 4 to $8 \mathrm{ml}$.). One aliquot was taken for phenylhydrazine assay, and another for carbon ${ }^{24}$ assay. The specific activity of the cortisone expressed in counts per minute per microgram was then determined from the radioactivity value and the quantitative cortisone value as determined by the phenylhydrazine assay. The quantity of cortisone in the plasma sample was calculated as previously described (2).

Procedure for determination of cortisone metabolites in urine. The method and reagents were the same as those previously described for hydrocortisone (1).

Studies with cortisone-4-C $C^{4}$. Cortisone-4-C ${ }^{n}$ acetate (0.49 mc. per $\mathrm{mM}$ ), hydrocortisone-4- $\mathrm{C}^{16}$ (1.47 mc. per $\mathrm{mM}$ ), tetrahydrocortisone-4- $\mathrm{C}^{\text {14 }}$ mono-acetate (0.4 mc. per $\mathrm{mM}$ ), and dihydrocortisone- $4-\mathrm{C}^{16}$ acetate $(0.4 \mathrm{mc}$. per mM) were made available through the Endocrine Study Section of the National Institutes of Health. ${ }^{2}$ The acetates were converted to the free steroids by hydroylsis at pH 7.4 with acetyl cholinesterase (Nutritional Bio-Chemicals Corp.), 500 units per $3 \mathrm{ml}$. $0.5 \mathrm{M}$ glycyl glycine buffer. The buffered solution was incubated at $37^{\circ} \mathrm{C}$. for

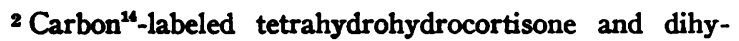
drohydrocortisone were prepared enzymatically from hydrocortisone-4-C $C^{14}$ (5).
18 hours, and then was extracted with dichloromethane. The dichloromethane was evaporated to dryness under nitrogen, and the residue chromatographed on paper for 18 hours (cyclohexane 2: benzene 4: methanol 4: water 1). The free cortisone, tetrahydrocortisone, and dihydrocortisone were eluted from the paper with 95 per cent ethanol. Reverse isotope dilution analysis, using paper chromatography, showed these to be essentially pure steroids.

The plasma and urine samples for radioactivity assay were extracted in the same manner as for the colorimetric assays; however, the dichloromethane extracts were transferred to planchets for counting.

The studies on the biliary excretion of the radiometabolites of cortisone-4-C $C^{14}$ were made on the same patient who was used for the hydrocortisone-4-C $C^{n}$ studies (1).

Procedure for carbon analysis of samples. All assays for carbon" were carried out with a Robinson (6) gasflow counter as previously described (1). Plasma, urine, and bile samples were counted as previously described (1).

\section{RESULTS}

Rate of disappearance of cortisone and "cortisonelike" material from plasma after intravenous administration

When the concentrations of phenylhydrazinereacting material present in the plasma after the infusion of cortisone were plotted against time on 
semilogarithmic paper, a straight line was obtained. (In a few of the subjects with cirrhosis, e.g., Figure 5, a single straight line was not observed.) In normal subjects the half-time of disappearance of the material reacting with phenylhydrazine was the same, irrespective of the quantity of cortisone administered over a range of 50 to $400 \mathrm{mg}$. This indicated that the rate of disappearance of cortisone (and its metabolites) was proportional to concentration over a wide range of concentrations. This permitted a comparison of the behavior of infused cortisone in different subjects under various conditions.

In 15 normal subjects, the mean biological halftime of the material reacting with phenylhydrazine was 60 minutes, with a range of 45 to $95 \mathrm{~min}$ utes (Figure 2). In patients with cirrhosis of the liver and myxedema, the biological half-times were not significantly prolonged. In the subjects with thyrotoxicosis, the half-times were shortened (mean, 28 minutes) and in the rheumatoid arthritis patients, the half-times were within the normal range.

In all groups except for the subjects with thyrotoxicosis, the differences in the half-times for the material reacting with phenylhydrazine were not greatly different from those in the normals, and thus it was not possible to be sure that cortisone per se was being metabolized at a different rate. Since both counter-current distribution and isotope dilution analysis of the material reacting with phenylhydrazine indicated that a large fraction of the steroid measured with the phenylhydrazine reagent was not cortisone, it was of interest to determine the rate of disappearance of true corti-

TABLE I

Disappearance of cortisone and phenylhydrazine-reacting steroids from plasma following intravenous administration of cortisone

\begin{tabular}{lcc}
\hline \hline & \multicolumn{2}{c}{ Biological half-times } \\
& (minutes) & \\
\cline { 2 - 3 } & $\begin{array}{c}\text { Phenyl- } \\
\text { hydrazine }\end{array}$ & Cortisone \\
\hline Normal & 55 & 23 \\
Normal & 50 & 30 \\
Normal & 60 & 35 \\
Normal & 55 & 25 \\
Cirrhosis & 60 & 30 \\
Cirrhosis & 70 & 30 \\
Cirrhosis & 60 & 30 \\
Myxedema & 60 & 25 \\
Thyrotoxicosis & 30 & 12 \\
\hline
\end{tabular}

sone in these groups (Table I). In four normal subjects, cortisone, as determined by isotope dilution assay, was found to disappear at a rate approximately twice that of the material reacting with phenylhydrazine. In three patients with cirrhosis of the liver and in one patient with myxedema, cortisone was also found to disappear at the normal rate. However, in one patient with thyrotoxicosis, the half-time for true cortisone (12 minutes) and the steroids reacting with phenylhydrazine (30 minutes) were both much reduced. In all cases the actual cortisone disappeared at approximately twice the rate of the phenylhydrazinereacting material.

\section{Disappearance of radioactive cortisone from plasma}

Following intravenous infusion of cortisone-4$\mathrm{C}^{\mathbf{1 4}}$, the concentration in plasma of isotopic substances extractable with dichloromethane decreased

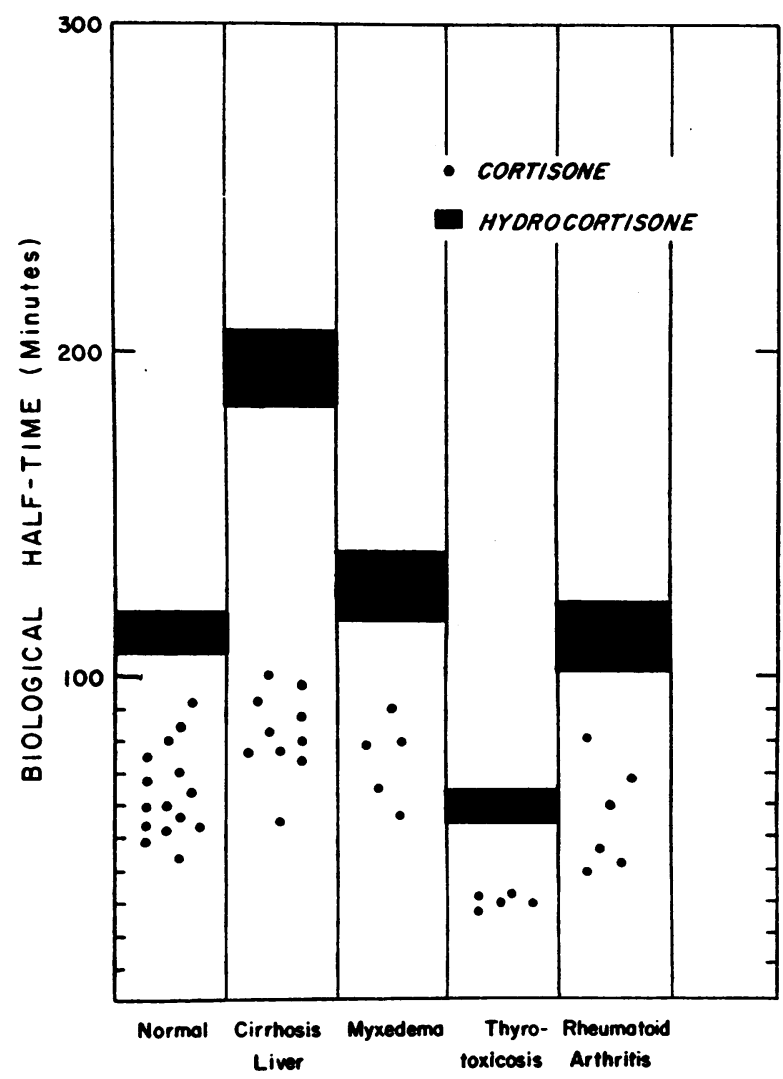

Fig. 2. Biological Half-Tmas of Steroms Reacting with Phenylhydrazine Following Intravenous. Administration of Hydrocortisone aNd CoRtisone 


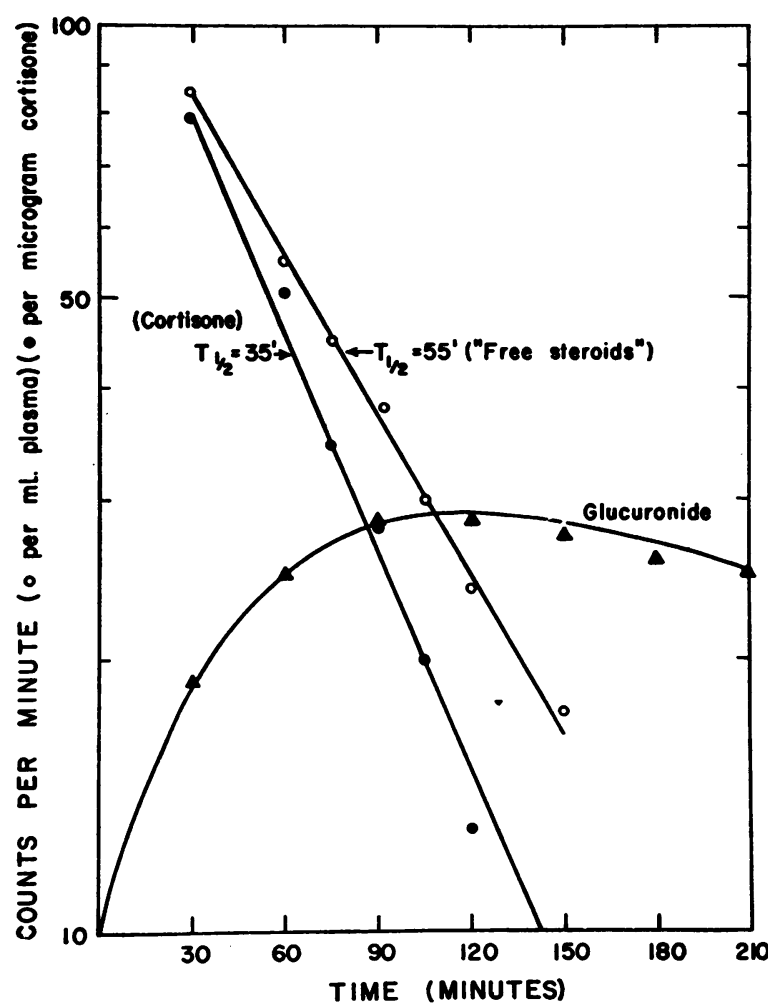

Fig. 3. Cortisone and Free and Glucuronide-Conjugated Steroids in Plasma After Intravenous AdMinistration OF CoRTisone-4-C ${ }^{14}$

logarithmically with time (Figure 3 ). In three subjects, half-times of 35,46 , and 55 minutes were obtained. A large fraction of the extractable material was not cortisone, and in two subjects the results of paper chromatographic analyses of plasma extracts obtained 30 to 120 minutes after infusion indicated that more than 60 per cent of the radioactivity of this fraction was attributable to the presence of other steroid products. Also, reverse isotope dilution analyses of the plasma samples in one subject revealed that the actual cortisone-4-C ${ }^{14}$ had a half-time of 35 minutes, whereas the half-time of the carbon ${ }^{14}$ was 55 minutes. On the basis of isotopic dilution studies of plasma after infusion of cortisone (e.g., Figure 4 ), it is reasonable to assume that hydrocortisone, tetrahydrocortisone, and tetrahydrohydrocortisone make up a large portion of these unconjugated radioactive metabolites.

In addition to the unconjugated metabolites in plasma, there was present a fraction of labeled metabolites that was rendered dichloromethane soluble following $\beta$-glucuronidase hydrolysis. As the free steroids disappeared from plasma, conjugated derivatives hydrolyzable with $\beta$-glucuronidase appeared, and reached a maximum at 90 to 120 minutes. At 90 minutes the free and conjugated fractions were of equal magnitude. Thereafter, the conjugated fraction exceeded the level of the free, and disappeared from the plasma at a rate slower than that of the free fractions.

\section{Comparative rates of disappearance from plasma of steroids structurally related to cortisone}

Table II shows the rates of disappearance from the plasma of several steroids structurally similar to cortisone, in a normal subject and in one patient with cirrhosis of the liver. The phenylhydrazine method was used for assay of all of these steroids except cortisone, and with all the steroids, except for cortisone, the assay method has been found to be relatively specific (on the basis of evaluation by isotope dilution assay). In the normal subject and the patient with cirrhosis, cortisone and the dihydro and tetrahydro derivatives of cortisone and hydrocortisone disappear much more rapidly than hydrocortisone.

\section{In vivo conversion of cortisone to hydrocortisone}

Cortisone was infused over a period of $12 \mathrm{~min}$ utes into a normal subject and a patient with cirrhosis of the liver, and blood samples were collected at various times after the termination of the infusion. Endogenous adrenal hydrocortisone production was suppressed by the prior administration of $\Delta^{1}, 9 \alpha$-fluorohydrocortisone $(2.5 \mathrm{mg}$. per day). The plasma samples were assayed for total

TABLE II

Biological half-times of various steroids following their intravenous administration

\begin{tabular}{lcc}
\hline \hline & \multicolumn{2}{c}{$\begin{array}{c}\text { Biological half-times } \\
\text { (minutes) }\end{array}$} \\
\cline { 2 - 3 } & Normal & Cirrhosis \\
\hline Hydrocortisone & 98 & 320 \\
Cortisone* & 23 & 30 \\
Dihydrocortisone & 25 & 32 \\
Tetrahydrocortisone & 27 & 25 \\
Dihydrohydrocortisone & 25 & 25 \\
Tetrahydrohydrocortisone & 23 & 27
\end{tabular}

* Cortisone was determined by the isotope dilution procedure, and all other steroids by the phenylhydrazine method. 


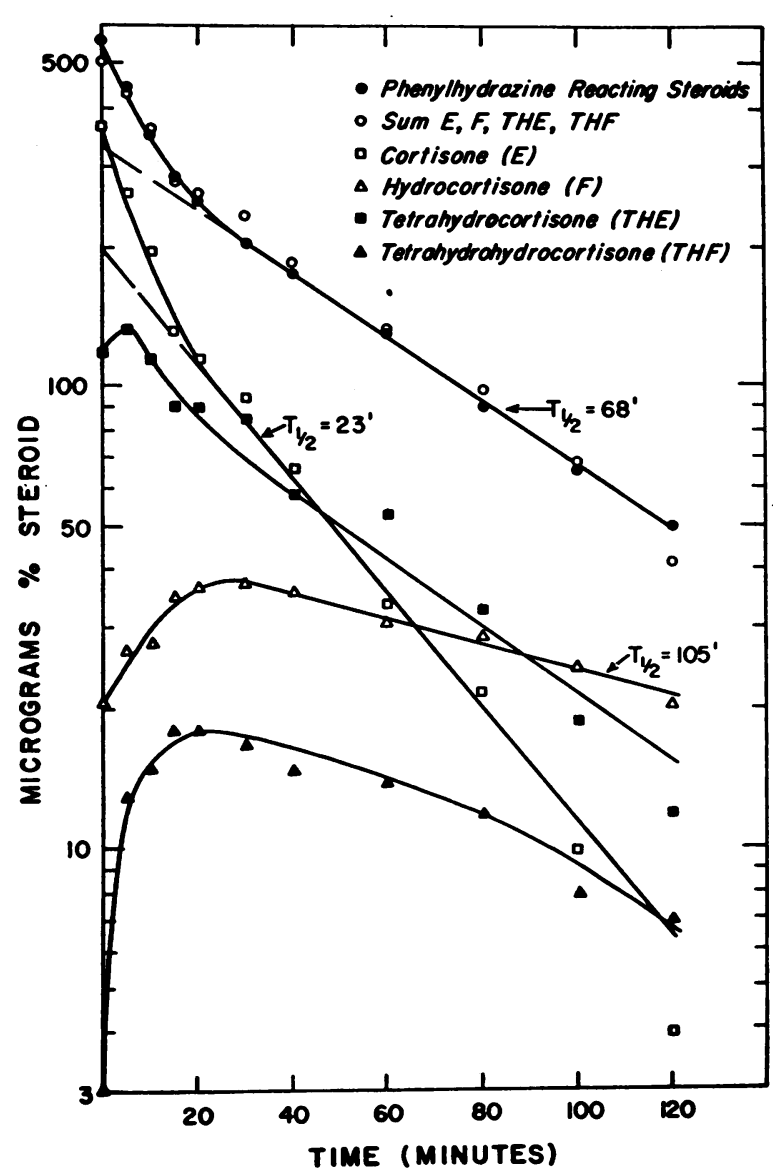

Fig. 4. Free Plasma Steroids After Infusion of 400 mG. Cortisone Into Normal Subject

material reacting with phenylhydrazine. With the use of the isotope dilution procedure with paper chromatography, the samples were assayed for hydrocortisone, cortisone, tetrahydrocortisone, and tetrahydrohydrocortisone. ${ }^{3}$ In the normal subject, one-half of the cortisone had disappeared in 23 minutes. Within one hour, the concentration of hydrocortisone and of tetrahydrocortisone equaled the level of cortisone, and after two hours the cortisone could no longer be detected (Figure 4). Figure 5 shows the results obtained in the

8 The accuracy of the isotope dilution assay was not in every instance checked by purification of the samples to a constant specific activity. In many assays, however, the samples were either re-chromatographed in different solvent systems or converted to their respective acetates and re-chromatographed. Whenever these additional purifications were carried out, the repeat specific activities checked with the first assay to within the average error of the method \pm 10 per cent. patient with cirrhosis of the liver. Cortisone disappeared at a normal rate, and after two hours the level of the material reacting with phenylhydrazine approximated the level of free hydrocortisone.

Three hundred mg. of carrier cortisone plus 3 microcuries of cortisone-4-C ${ }^{14}$ were infused into a normal subject over a period of 10 minutes. Endogenous hydrocortisone production was suppressed by the prior administration of $\Delta^{1}, 9 \alpha$-fluorohydrocortisone. Blood samples were collected every 30 minutes during the two-hour period following termination of the infusion. Each plasma sample was extracted with dichloromethane and subjected to paper chromatography, and the cortisone and hydrocortisone eluted from the paper with 95 per cent ethanol. The specific activity of each steroid was determined. All samples of cortisone and hydrocortisone gave similar $( \pm 10$ per cent) specific activities. With labeled hydrocortisone, it was not possible to detect any conversion of hydrocortisone to cortisone."

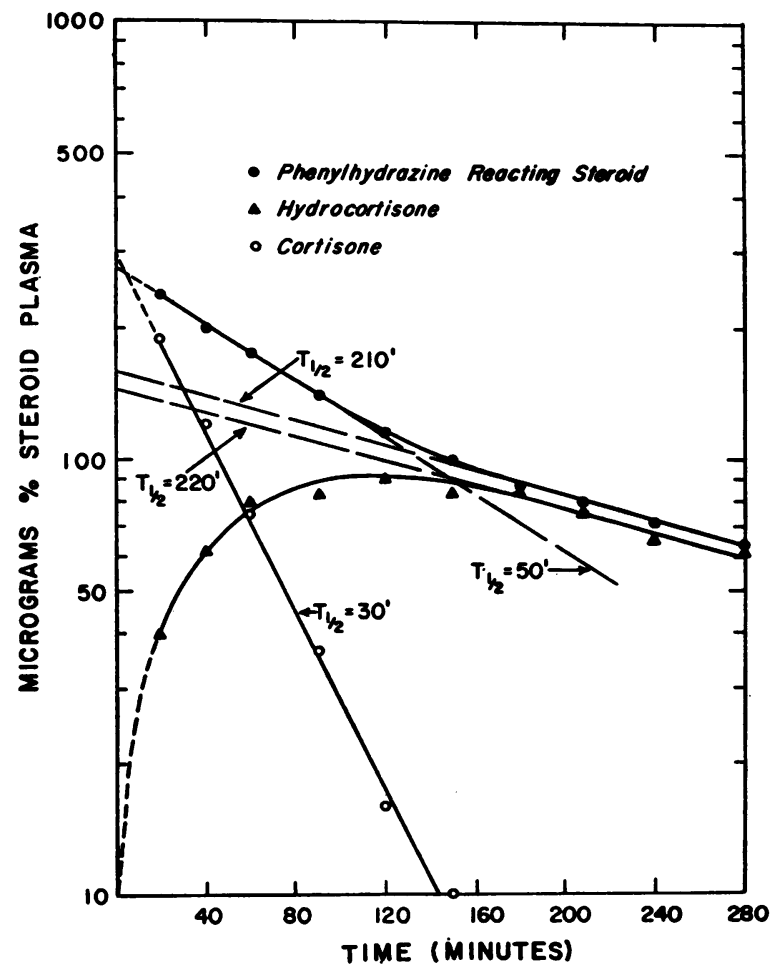

Fig. 5. Free Plasma Steroms After Infusion of 200 mg. Cortisone Into Patient with Cirrhosis of Liver

4 Some conversion of hydrocortisone to cortisone was shown to occur, however, as was evident from the isolation of $\mathrm{C}^{4}$-labeled cortisone from the urine, after administration of $\mathrm{C}^{14}$-labeled hydrocortisone. 
TABLE III

Plasma steroid concentration following administration of cortisone or hydrocortisone, and cortisone or hydrocortisone acetate

\begin{tabular}{|c|c|c|c|c|c|}
\hline & \multicolumn{5}{|c|}{ Minutes following administration of steroid } \\
\hline & 30 & 60 & 90 & 120 & 180 \\
\hline $\begin{array}{l}\text { Cortisone, } 200 \mathrm{mg} \text {. intravenously } \\
\text { PNH* } \\
\text { E } \\
\text { F } \\
\text { Hydrocortisone, } 200 \mathrm{mg} \text {. intravenously }\end{array}$ & $\begin{array}{r}120 \dagger \\
75 \\
38 \\
160\end{array}$ & $\begin{array}{r}86 \\
33 \\
32 \\
135\end{array}$ & $\begin{array}{r}53 \\
14 \\
25 \\
120\end{array}$ & $\begin{array}{r}42 \\
6 \\
32 \\
100\end{array}$ & $\begin{array}{r}20 \\
0 \\
14 \\
71\end{array}$ \\
\hline $\begin{array}{l}\text { Cortisone, } 200 \mathrm{mg} \text {. orally } \\
\text { PNH } \\
\underset{\text { E }}{\text { F }} \\
\text { Hydrocortisone, } 200 \mathrm{mg} \text {. orally }\end{array}$ & $\begin{array}{l}87 \\
20 \\
70 \\
95\end{array}$ & $\begin{array}{r}160 \\
14 \\
150 \\
200\end{array}$ & $\begin{array}{r}180 \\
6 \\
160 \\
220\end{array}$ & $\begin{array}{r}140 \\
3 \\
140 \\
180\end{array}$ & $\begin{array}{r}96 \\
0 \\
90 \\
140\end{array}$ \\
\hline $\begin{array}{l}\text { Cortisone, } 200 \mathrm{mg} \text {. intramuscularly } \\
\text { PNH } \\
\underset{\text { F }}{\text { Hydrocortisone, } 200 \mathrm{mg} \text {. intramuscularly }}\end{array}$ & $\begin{array}{r}33 \\
3 \\
30 \\
30\end{array}$ & $\begin{array}{r}35 \\
2 \\
33 \\
48\end{array}$ & $\begin{array}{r}35 \\
2 \\
36 \\
55\end{array}$ & $\begin{array}{r}33 \\
0 \\
35 \\
50\end{array}$ & $\begin{array}{r}30 \\
0 \\
30 \\
45\end{array}$ \\
\hline $\begin{array}{l}\text { Cortisone acetate, } 225 \mathrm{mg} \text {. orally } \\
\text { PNH } \\
\text { F } \\
\text { Hydrocortisone acetate, } 225 \mathrm{mg} \text {. orally }\end{array}$ & $\begin{array}{r}35 \\
2 \\
32 \\
8\end{array}$ & $\begin{array}{r}37 \\
3 \\
36 \\
10\end{array}$ & $\begin{array}{r}40 \\
3 \\
40 \\
16\end{array}$ & $\begin{array}{r}40 \\
2 \\
35 \\
16\end{array}$ & $\begin{array}{r}35 \\
0 \\
32 \\
8\end{array}$ \\
\hline
\end{tabular}

* $\mathrm{PNH}=$ Phenylhydrazine-reacting steroids $; \mathrm{E}=$ Cortisone $; \mathrm{F}=$ Hydrocortisone.

t Figures are expressed as micrograms per cent.

Table III indicates the extent of the in vivo conversion of cortisone to hydrocortisone following the administration via different routes of cortisone and hydrocortisone to a normal subject in whom adrenal hydrocortisone production had been suppressed by the prior administration of $\Delta^{1}, 9 \alpha-$ fluorohydrocortisone. Two hundred mg. of crystalline cortisone and hydrocortisone and $225 \mu \mathrm{g}$. of the acetates were administered. Following the administration of cortisone, both cortisone and hydrocortisone were assayed by isotope dilution. Following the administration of hydrocortisone, the hydrocortisone was assayed by the phenylhydrazine method. ${ }^{5}$ After oral or intramuscular administration of cortisone, the plasma hydrocortisone levels were one-half to two-thirds the levels obtained after the administration of comparable

5 This assay method has been shown to be highly specific for hydrocortisone under these conditions. After oral or intramuscular administration of hydrocortisone or hydrocortisone acetate, more than 90 per cent of the steroid reacting with phenylhydrazine was hydrocortisone, and after intravenous administration of hydrocortisone, 80 to 90 per cent of the steroid reacting with phenylhydrazine was found to be hydrocortisone. amounts of hydrocortisone. The higher plasma hydrocortisone levels after oral cortisone acetate as contrasted with oral hydrocortisone acetate are the result of the incomplete absorption of hydrocortisone acetate (unpublished observations). Following intravenous administration of cortisone, lower hydrocortisone levels are obtained, presumably because via this route of administration a larger fraction of the cortisone is rapidly transformed to tetrahydrocortisone.

\section{Excretion of cortisone and metabolites}

Following intravenous administration of cortisone, 30 to 40 per cent of the administered steroid was recovered as material reacting with phenylhydrazine in the dichloromethane extract of glucuronidase-treated urine during the first 24 hours. Approximately one-half of the recovered material appeared in the urine during the first four hours. Direct extraction of the urine with dichloromethane yielded phenylhydrazine substances equivalent to 3 to 7 per cent of the administered dose, and most of these unconjugated steroids were excreted during the first six hours. 
TABLE IV

Urinary axcrotion of phenylhydrazime-reacting steroids following infusion of $100 \mathrm{mg}$. cortisone

\begin{tabular}{|c|c|c|}
\hline & \multicolumn{2}{|c|}{$\begin{array}{l}\text { Urinary steroids* } \\
(\%)\end{array}$} \\
\hline & Free & Glucuronide \\
\hline $\begin{array}{l}\text { Cortisone } \\
\text { Hydrocortisone } \\
\text { Dihydrocortisone } \\
\text { Tetrahydrocortisone } \\
\text { Dihydrohydrocortisone } \\
\text { Tetrahydrohydrocortisone } \\
\text { Phenylhydrazine assay } \\
\text { Carbon } 14\end{array}$ & $\begin{array}{l}0.30 \\
0.60 \\
0.04 \\
0.20 \\
0.01 \\
0.08 \\
3.00 \\
6.00\end{array}$ & $\begin{array}{r}20.0 \\
6.0 \\
28.0 \\
52.0\end{array}$ \\
\hline
\end{tabular}

* Individual steroids determined by the isotope dilution method.

I Independent study on this same normal subject given cortisone-4-C ${ }^{14}$.

Hydrocortisone represented only one of the pathways of metabolism of cortisone. A large fraction of the cortisone was rapidly converted. to tetrahydrocortisone. Table IV indicates the portion of the steroids reacting to phenylhydrazine that were made up of free cortisone, hydrocortisone, and their dihydro and tetrahydro derivatives, following the infusion of $100 \mathrm{mg}$. of cortisone. These figures were obtained by isotope dilution assay. Most of the glucuronide conjugated metabolites reacting with phenylhydrazine were compounds more polar than cortisone, and more than seventy per cent of the steroid glucuronides reacting with phenylhydrazine consisted of tetrahydrocortisone.

\section{Urinary excretion of radiometabolites of cortisone}

Figure 6 shows the cumulative urinary excretion of labeled cortisone and its metabolites following intravenous administration of a tracer quantity of cortisone-4-C $\mathrm{C}^{14}$ plus $200 \mathrm{mg}$. carrier cortisone. Eighty per cent of the injected dose was excreted within the first 24 hours. A small additional quantity appeared during the second, and a trace during the third 24-hour period. By the fourth day, no labeled urinary products could be demonstrated. In three such studies, an average of 91 per cent of the infused radioactivity was accounted for through urinary excretion. The half-time of the initial excretion rate of these products averaged 3.6 hours. During the first six hours, there was excreted a urinary fraction which was extractable with dichloromethane without

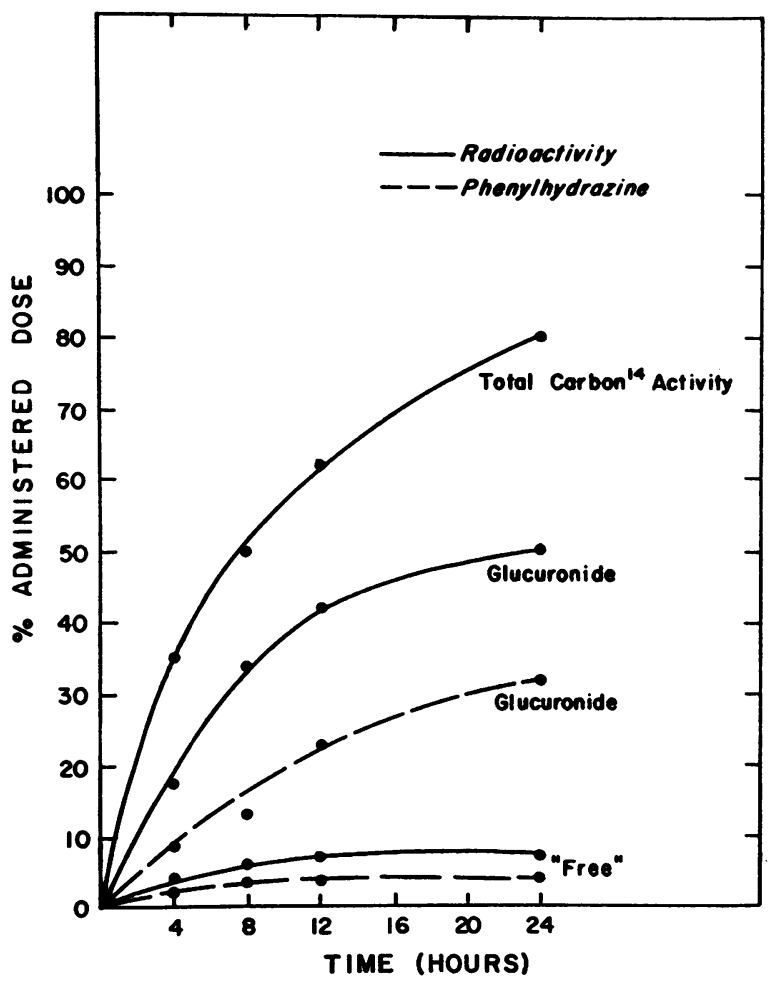

Fig. 6. Urinary Excretion (Cumulative) of Radionctivity aNd Steroids Reacting with PhenylhydRazine Following Infusion of $1.1 \mu \mathrm{C}$. Cortisone-4-C and 200 mg. Carrter Cortisone

prior hydrolysis. This fraction contributed to seven per cent of the total infused radioactivity, whereas four per cent of the administered steroid could be recovered as material reacting with phenylhydrazine. Following glucuronidase hydrolysis and dichloromethane extraction of the urine, 55 per cent of the administered radioactivity was recovered, but only 30 per cent as measured by the phenylhydrazine assay.

\section{Biliary excretion of radiometabolites of cortisone}

Following intravenous administration of cortisone-4-C $\mathrm{C}^{14}$ to a patient with a biliary cannula (1), the rate of accumulation of metabolites in the urine and the total quantity appearing were much the same as in the normals. The total quantity of metabolites appearing in the bile was four per cent of the injected dose. Analysis of the feces collected for three days following the infusion of the steroid failed to yield any radioactivity. Direct dichloromethane extraction of the bile failed to reveal the presence of any radioactivity. 


\section{Absorption of cortisone}

Following oral administration, as with intravenous infusion, 30 to 40 per cent of the administered steroid was recovered in the urine as metabolites of cortisone reacting with phenylhydrazine within the first 24 hours. Following oral administration of either a trace quantity of cortisone$4-\mathrm{C}^{14}$ or cortisone-4-C ${ }^{14}$ plus $200 \mathrm{mg}$. added carrier, a cumulative rate of excretion of labeled metabolites similar to that obtained after intravenous administration was found.

\section{DISCUSSION}

These studies point up the importance of evaluating the specificity of the assay procedure in studies of steroid metabolism. Determinations of the rate of metabolism of cortisone, as judged by its disappearance from the plasma following intravenous infusion, through the use of the phenylhydrazine assay method, indicated an average biological half-time in normal subjects of about 60 minutes. However, by means of a specific isotope dilution method, it was found that the actual cortisone had an average plasma biological halftime of 28 minutes. It was shown that the phenylhydrazine method measured the combined rates of disappearance of cortisone and metabolites of cortisone (hydrocortisone, tetrahydrohydrocortisone, and tetrahydrocortisone). The disappearance of true cortisone, as determined by an isotope dilution assay, was found in all cases to have the characteristics of a first-order reaction. Since less than one per cent of the administered cortisone was excreted as unaltered steroid, the rate of disappearance of cortisone from plasma was presumably a measure of its rate of metabolism.

In patients with liver disease, cortisone disappeared from the plasma at the normal rate, whereas hydrocortisone has been shown to be metabolized at a much slower rate $(1,7)$. In patients with liver disease, cortisone was metabolized at the same rapid rate as the biologically inactive dihydro and tetrahydro metabolites of cortisone and hydrocortisone.

Patients with thyrotoxicosis showed an increased rate of metabolism of both cortisone and hydrocortisone. Corticosterone has also been found to be metabolized at an increased rate in thyrotoxicosis (8). This may indicate that in thyrotoxicosis there is a general increased turnover of the various steroid dihydro-dehydrogenase enzymes required for saturation of ring $A$ of many of the steroids.

These data on the metabolism of cortisone are at variance with those of Eik-Nes, Sandberg, Tyler, and Samuels (9), who, using a phenylhydrazine assay, reported that cortisone and hydrocortisone were metabolized at the same rate in normal subjects.

Cortisone was metabolized at a rate approximately four times faster than that of hydrocortisone; however, cortisone has approximately twothirds the biological activity of hydrocortisone. This indicates a clear lack of correlation between rate of metabolism of a steroid known to be biologically potent and its biological activity as observed in man and animals. ${ }^{\circ}$ Since, however, such a large fraction of the administered cortisone was found to be rapidly converted to hydrocortisone, it may be that most of the biological activity of cortisone resides in that fraction that is converted to hydrocortisone.

Previous investigators have shown by perfusion of the liver $(11,12)$ and with liver slices $(13$, $14)$ and liver homogenates $(15,16)$, that cortisone is converted to hydrocortisone. Also, studies of urinary steroid metabolites in man following the administration of cortisone have demonstrated this conversion (17-19). Attempts to evaluate the extent of the conversion of cortisone to hydrocortisone through studies of the levels of cortisone and hydrocortisone in the urine may give an incomplete picture, since only a small fraction of the administered steroid is excreted as cortisone and hydrocortisone. Studies of plasma levels give a better indication of the initial sequence of metabolic transformations of administered steroid.

Our studies demonstrated that a large fraction of the administered cortisone was converted to hydrocortisone, and the relative ratio of hydrocortisone to cortisone in the plasma after administration of cortisone was very much dependent on the method of administration. Following a rapid infusion of a large amount of cortisone, a large fraction of the cortisone was converted initially to

\footnotetext{
- $9 \alpha$-fluorohydrocortisone, a steroid possessing considerably more biological activity than hydrocortisone, has been found to be metabolized at a slightly more rapid rate than hydrocortisone (unpublished observations [10]).
} 
tetrahydrocortisone. After the oral or intramuscular administration of cortisone, hydrocortisone made up the largest fraction of phenylhydrazine steroid in the plasma, and following orally administered cortisone acetate with its slower absorption, hydrocortisone accounted for 90 to 95 per cent of the plasma steroid reacting with phenylhydrazine. Thus, with the usual routes of administration of cortisone as it is used therapeutically, the plasma levels of hydrocortisone were one-half to two-thirds those obtained after administration of comparable amounts of hydrocortisone. This difference cannot be explained on the basis of a more incomplete absorption of the orally administered cortisone (Figure 6). Cortisone acetate, like cortisone and hydrocortisone, has been found to be completely absorbed after oral administration, but at a slightly slower rate. The lower plasma hydrocortisone levels after orally administered hydrocortisone acetate are the result of its incomplete absorption.

Clinical studies on the relative potency of cortisone versus hydrocortisone administered orally to humans have shown that, on a weight basis, cortisone is approximately two-thirds as effective as hydrocortisone as an anti-inflammatory agent (20, 21). Cortisone administered orally also appears to be about two-thirds as effective as hydrocortisone in suppression of the adrenal (22). In addition, studies on the relative potency of cortisone and hydrocortisone in the rat, through the use of such indices as thymic involution, liver glycogen deposition, and the muscle work test, have shown that cortisone is only about two-thirds as active as hydrocortisone (23). Thus, by several criteria, cortisone has been shown to be biologically less active than hydrocortisone. From our observations it would appear that one could account for most, if not all, of the biological activity of cortisone on the basis of that fraction that is converted to hydrocortisone (plasma levels of hydrocortisone after cortisone, one-half to two-thirds the levels of hydrocortisone after comparable doses of hydrocortisone). Intra-articularly injected cortisone or its acetate, though reported to be converted in part to hydrocortisone (24), has been found to be very much less effective in the relief of joint symptoms than either hydrocortisone or its acetate (25-27). This lack of effectiveness may be due to the failure of the synovium to effect any sig- nificant conversion of cortisone to hydrocortisone. Indeed, preliminary studies have shown no detectable conversion of cortisone to hydrocortisone when cortisone was injected into the intra-articular space in patients with active rheumatoid arthritis (28).

The liver would appear to be the organ responsible for the conversion of cortisone to hydrocortisone, and Ingle (29) has stated that cortisone is biologically inactive for maintenance of life in the hepatectomized, adrenalectomized rat, but not in rats with an intact liver. Subsequent studies, however, by Ingle and his co-workers $(30,31)$ have demonstrated by other physiological functions that cortisone was active in the absence of the liver. In these studies, no attempt was made to determine if the extra-hepatic tissues could transform cortisone to hydrocortisone. Steroid glucocorticoid properties (glycogen deposition, pituitary-adrenal suppression, anti-inflammatory activity) may require the presence of an $11 \beta$-hydroxy function. The glucocorticoid properties of the 11-dehydro steroids may depend on the presence of liver $11 \beta$ hydroxy dehydrogenase enzymes. Preliminary unpublished data on the metabolism of $\Delta^{\mathbf{1}}$-cortisone have shown that following oral administration of this steroid, the plasma levels of $\Delta^{1}$-hydrocortisone far exceed the $\Delta^{1}$-cortisone levels. This again suggests that the biological activity of the administered 11-dehydro compound ( $\Delta^{1}$-cortisone) may reside in that fraction that is transformed to $\Delta^{1}$ hydrocortisone. It is also of interest to note that the 2-methyl analogues of cortisone and $9 \alpha$-fluorohydrocortisone have very much less glucocorticoid activity than the parent $11 \beta$-hydroxy steroids (32). It will be of interest to determine if the liver in vivo is unable to effect a reduction of the 11-keto groups of the 2-methyl steroids.

The major route of excretion of labeled metabolites of cortisone was via the urine, in which up to 91 per cent of the administered carbon ${ }^{14}$ appeared in 72 hours. Only a minor fraction of the steroid (about four per cent) was excreted into the gastrointestinal tract via the bile. Previous results obtained with hydrocortisone were similar $(1,33$, 34). Studies with corticosterone have yielded similar results; however, slightly more is excreted through the bile and less via the urine $(8,35)$.

In the present study, recoveries of metabolites in urine indicated that three to seven per cent of the 
cortisone, when administered either orally or intravenously, was directly extractable with dichloromethane without prior hydrolysis. All of these freely extractable steroids appeared within the first six to eight hours after administration. Only 0.3 per cent of the administered steroid was excreted as unaltered cortisone, and the remaining steroids consisted for the most part of hydrocortisone, tetrahydrocortisone, and tetrahydrohydrocortisone.

Urinary metabolites equivalent to 50 to 60 per cent of the administered cortisone-4-C $\mathrm{C}^{14}$ were released by enzymatic hydrolysis ( $\beta$-glucuronidase) as determined by radioactivity analysis. Thirty to 40 per cent of the administered cortisone was accounted for in the urine as metabolites reacting with phenylhydrazine.

These data agree well with previously published reports on cortisone metabolites extractable with butanol (36), or extractable with chloroform or similar solvents following $\beta$-glucuronidase hydrolysis $(37,38)$. The disparity between the carbon $^{14}$ and phenylhydrazine analyses may be in large measure accounted for in the same way as with the hydrocortisone metabolites (39)-the presence of $\mathrm{C}_{20}$-hydroxy compounds that do not react with phenylhydrazine. Also, a small fraction of the non-phenylhydrazine reacting material may be $\mathrm{C}_{19}-17$ ketosteroids. Tetrahydrocortisone made up the major portion of the metabolites reacting with phenylhydrazine, and tetrahydrocortisone and tetrahydrohydrocortisone accounted for 93 per cent of all the phenylhydrazine-reactive giucuronide conjugates.?

After prior hydrolysis of the conjugated prod-

7 The following unpublished data may be pertinent to the findings that following intravenous administration of cortisone, the urinary concentration of tetrahydrocortisone glucuronide greatly exceeds the concentration of tetrahydrohydrocortisone glucuronide: Following intravenous infusion of tetrahydrocortisone, seven per cent was recovered in the urine as tetrahydrohydrocortisone, and 40 per cent as tetrahydrocortisone after glucuronidase hydrolysis. Twenty per cent of infused tetrahydrohydrocortisone was recovered in the urine as tetrahydrocortisone, and 30 per cent as tetrahydrohydrocortisone after glucuronidase hydrolysis. Thus, the presence of the $\alpha, \beta$ unsaturated 3-keto group in ring A seems to direct the enzymatic reduction of cortisone $\rightarrow$ hydrocortisone, whereas the saturated ring $A$ seems to favor oxidation of the $11 \beta$ hydroxy group (tetrahydrohydrocortisone $\rightarrow$ tetrahydrocortisone). ucts with $\beta$-glucuronidase and extraction of released steroids with dichloromethane, an additional 5 to 10 per cent of the infused isotope can be extracted with ethyl acetate, and another 10 to 20 per cent with $\mathrm{n}$-butanol saturated with water. Whether these fractions represent glucuronides hydrolyzable but not extracted with dichloromethane, glucuronides resisting hydrolysis with bacterial enzymes, or sulfates or other conjugation products, remains to be determined, as does the nature of the metabolites in the urine residue completely resisting extraction (five to ten per cent). Acid hydrolysis at room temperature for 24 hours will not make this latter fraction soluble in organic solvents.

\section{SUMMARY}

The physiological disposition and metabolic fate of cortisone were studied in man following intravenous infusion of steroid in doses up to $400 \mathrm{mg}$. In normal subjects, the biological half-life of the steroids reacting with phenylhydrazine in the plasma after infusion of cortisone was shown to average 60 minutes. Following infusion of tracer quantities of cortisone-4-C $\mathrm{C}^{14}$, the biological half-life of the unconjugated radioactive metabolites was found to be 35 to 55 minutes. A large fraction of these steroids measured with the phenylhydrazine reagent and by radioactive assay was, however, not cortisone. The biological half-life of the actual cortisone was found by a specific isotope dilution assay procedure to average 28 minutes in normal subjects. In patients with thyrotoxicosis, cortisone was found to disappear from the plasma at an increased rate, and in patients with liver disease, cortisone disappeared at the normal rate.

Administered cortisone was found to be very rapidly metabolized to hydrocortisone. The plasma hydrocortisone levels after oral or intramuscular administration of cortisone were found to be one-half to two-thirds those found following administration of comparable amounts of hydrocortisone. The absorption of cortisone from the gastrointestinal tract was found to be both rapid and complete. It was suggested that most, if not all, of the biological activity of cortisone could be accounted for by that fraction that was transformed to hydrocortisone.

Normal subjects excreted approximately 90 per cent of the administered steroid as urinary metabo- 
lites in 72 hours, as judged from excretion of carbon ${ }^{14}$ following infusion of tracer or larger quantities of labeled steroid. Four per cent was excreted in the bile. Three to seven per cent was excreted in the urine as unconjugated substances; 0.3 per cent as unchanged cortisone, and another 0.6 per cent as hydrocortisone. About 55 per cent of the administered steroid was exereted as metabolites hydrolyzable with bacterial $\beta$-glucuronidase; however, only about one-half of this fraction was detectable with the phenylhydrazine reaction, and tetrahydrocortisone was found to make up 70 per cent of this latter fraction.

\section{ACKNOWLEDGMENT}

We are indebted to the technical assistance of Serafim L. Guerra, Miss Aurora Karrer, and Miss Margaret Ballier. The generous supplies of steroid were kindly supplied by Merck and Company and by the Upjohn Company.

\section{REFERENCES}

1. Peterson, R. E., Wyngaarden, J. B., Guerra, S. L., Brodie, B. B., and Bunim, J. J., The physiological disposition and metabolic fate of hydrocortisone in man. J. Clin. Invest., 1955, 34, 1779.

2. Peterson, R. E., Karrer, A., and Guerra, S. L., Evaluation of the Silber-Porter procedure for determination of plasma hydrocortisone. Anal. Chem., 1957, 29, 144.

3. Silber, R. H., and Porter, C. C., The determination of 17,21-dihydroxy-20-ketosteroids in urine and plasma. J. Biol. Chem., 1954, 210, 923.

4. Bush, I. E., Methods of paper chromatography of steroids applicable to the study of steroids in mammalian blood and tissues. Biochem. J., 1952, 50, 370.

5. Tomkins, G. M., Enzymatic mechanisms of hormone metabolism. I. Oxidation-reduction of the steroid nucleus. Recent Progress in Hormone Research, vol. 12. New York, Academic Press, 1956, p. 125.

6. Robinson, C. V., Windowless, flow-type proportional counter for counting $C^{14}$. Science, 1950, 112, 198.

7. Brown, H., Willardson, D. G., Samuels, L. T., and Tyler, F. H., 17-hydroxycorticosteroid metabolism in liver disease. J. Clin. Invest., 1954, 33, 1524.

8. Peterson, R. E., The physiological disposition and metabolic fate of corticosterone in man, In preparation.

9. Eik-Nes, K., Sandberg, A. A., Tyler, F. H., and Samuels, L. T., Plasma levels of 17-hydroxycorticosteroids following the administration of adrenal steroids. Acta endocrinol., 1955, 18, 244.

10. Peterson, R. E., Metabolism of some of the newer synthetic steroids, Unpublished observations.

11. Hechter, O., Solomon, M. M., and Caspi, E., Corti- costenoid metabolism in liver: Studies on perfused rat livere. Endecrinology, 1953, 53, 202.

12. Caspio, E, Y., Levy, H., and Hechter, O. M., Cortisone metabolism in liver. II. Isolation of certain cortisone metabolites. Anch. Biochem. \& Biophys., 1953, 45, 169.

13. Schneider, J. J, and Horstmann, R. M., Effects of incubating compound $E$ and related steroids with various surviving rat tissues. J. Biol. Chen., 1952, 196, 629.

14. Eisenstein, A. B., Steroid compounds resulting from incubation of cortisone with surviving liver slices. Proc. Soc. Exper. Biol. \& Med., 1953, 83, 27.

15. Fish, C. A., Hayano, M., and Pincus, G., Conversion of cortisone to 17-hydro-corticasterone by liver homogenates. Arch. Biochein \& Biophys., 1953, 42 , 480.

16. Amelung, D., Hübener, H. J., Rokn, L., and Meyerheim, G., Conversion of cortisone to compound F. J. Clin. Endocrinol. \& Metab., 1953, 13, 1125.

17. Cope, C. L., and Hurlock, B., Same aspects of adrenal cortical metabolism. Clin. Sc., 1954, 13, 69.

18. Burstein, S., Savard, K., and Dorfman, R. I., The in vivo metabolism of cortisone. Endocrinology, 1953, 52, 448.

19. Burton, R. B., Keutmann, E. H., and Waterhouse, C., The conversion of cortisone acetate to other alphaketolic steroids. J. Clin. Endocrinol. \& Metab., 1953, 13, 48.

20. Ward, L. E., Polley, H. F., Slocumb, C. H., and Hench, P. S., Comparative effects of hydrocortisone, hydrocortisone acetate, and cortisone acetate in rheumatoid arthritis. J. Lab. \& Clin. Med., 1952, 40, 959.

21. Boland, E. W., Antirheumatic effects of hydrocortisone (free alcohol), hydrocortisone acetate, and cortisone (free alcohol), as compared with cortisone acetate; results from oral administration in patients with rheumatoid arthritis. Brit. M. J., 1952, 1, 559.

22. Kupperman, H. S., Blatt, M. H. G., Vessell, M., Gagliani, J., Weisbader, H., and Vosburgh, L., The comparative effects of metacortandracin, $9-\alpha-$ fluorohydrocortisone and hydrocortisone upon ACTH secretion in man. J. Clin. Endocrinol. \& Metab., 1955, 15, 911.

23. Stephenson, N. R., The relative potency of adrenal corticoids by the thymus involution method. Canad. J. Biochem. \& Physiol., 1956, 34, 253.

24. Wilson, H., Fairbanks, R., Scialabba, D., McEwen, C., and Ziff, M., Metabolites of hydrocortisone and cortisone in synovial fluid in rheumatoid arthritis. J. Clin. Endocrinol. \& Metab., 1956, 16, 86.

25. Hollander, J. L., Brown, E. M., Jr., Jessar, R. A., and Brown, C. Y., Hydrocortisone and cortisone injected into arthritic joints; comparative effects of and use of hydrocortisone as a local antiarthritic agent. J. A. M. A., 1951, 147, 1629. 
26. Ziff, M., Scull, E., Ford, D., McEwen, C., and Bunim, J. J., Effects in rheumatoid arthritis of hydrocortisone and cortisone injected intra-articularly. Arch. Int. Med., 1952, 90, 774.

27. Dixon, A. St. J., and Bywaters, E. G. L., The effect of intra-articular injection of cortisone acetate and of hydrocortisone acetate in rheumatoid arthritis. Clin. Sc., 1953, 12, 15.

28. Peterson, R. E., Unpublished observations.

29. Ingle, D., Adrenal Cortex, Third Josiah Macy, Jr. Foundation Conference, 1951, p. 107.

30. Ingle, D. J., Nezamis, J. E., and Morley; E. H., Effect of adrenal cortical extract, cortisone and hydrocortisone upon the work of the adrenalectomized-eviscerate rat. Endocrinology, 1953, 53, 582.

31. Bondy, P. K., Ingle, D. J., and Meeks, R. C., Influence of adrenal cortical hormones upon the level of plasma amino acids in eviscerate rats. Endocrinology, 1954, 55, 354.

32. Dulin, W. E., Bowman, B. J., and Stafford, R. O., Effects of 2-methylation on glucocorticoid activity of various C-21 steroids. Proc. Soc. Exper. Biol. \& Med., 1957, 94, 303.

33. Hellman, L., Bradlow, H. L., Adesman, J., Fukushima, D. K., Kulp, J. L., and Gallagher, T. F., The fate of hydrocortisone-4-C $\mathrm{C}^{14}$ in man. J. Clin. Invest., 1954, 33, 1106.
34. Migeon, C. J., Sandberg, A. A., Decker, H. A., Smith, D. F., Paul, A. C., and Samuels, L. T., Metabolism of 4-C ${ }^{14}$-cortisol in man: Body distribution and rates of conjugation. J. Clin. Endocrinol. \& Metab., 1956, 16, 1137.

35. Hellman, L., Bradlow, H. L., Frazell, E. L., and Gallagher, T. F., Tracer studies of the absorption and fate of steroid hormones in man. J. Clin. Invest., 1956, 35, 1033.

36. Reddy, W. J., Jenkins, D., and Thorn, G. W., Estimation of 17-hydroxycorticoids in urine. Metabolism, 1952, 1, 511.

37. Sandberg, A. A., Nelson, D. H., Glenn, E. M., Tyler, F. H., and Samuels, L. T., 17-hydroxycorticosteroids and 17-ketosteroids in urine of human subjects: Clinical application of a method employing $\beta$-glucuronidase hydrolysis. J. Clin. Endocrinol. \& Metab., 1953, 13, 1445.

38. Vestergaard, P., Investigations on the estimation of 17-hydroxycorticoids in urine using the Porter/ Silber reaction. Acta endocrinol., 1953, 13, 241.

39. Fukushima, D. K., Leeds, N. S., Bradlow, H. L., Kritchevsky, T. H., Stokem, M. B., and Gallagher, T. F., The characterization of four new metabolites of adrenocortical hormones. J. Biol. Chem., 1955, 212, 449. 